\title{
BMJ Open Impact of the urgent care telephone service NHS 111 pilot sites: a controlled before and after study
}

\author{
J Turner, A O'Cathain, E Knowles, J Nicholl
}

To cite: Turner J, O'Cathain A, Knowles E, et al. Impact of the urgent care telephone service NHS 111 pilot sites: a controlled before and after study. BMJ Open 2013:3:e003451.

doi:10.1136/bmjopen-2013003451

- Prepublication history and additional material for this paper is available online. To view these files please visit the journal online (http://dx.doi.org/10.1136/ bmjopen-2013-003451)

Received 20 June 2013 Accepted 25 September 2013

CrossMark Revised 23 August 2013

\section{ABSTRACT}

Objectives: To measure the impact of the urgent care telephone service NHS 111 on the emergency and urgent care system.

Design: Controlled before and after study using routine data.

Setting: Four pilot sites and three control sites covering a total population of 3.6 million in England, UK.

Participants and data: Routine data on 36 months of use of emergency ambulance service calls and incidents, emergency department attendances, urgent care contacts (general practice (GP) out of hours, walk in and urgent care centres) and calls to the telephone triage service NHS direct.

Intervention: NHS 111, a new 24 h 7 day a week telephone service for non-emergency health problems, operated by trained non-clinical call handlers with clinical support from nurse advisors, using NHS Pathways software to triage calls to different services and home care.

Main outcomes: Changes in use of emergency and urgent care services.

Results: NHS 111 triaged 277163 calls in the first year of operation for a population of 1.8 million. There was no change overall in emergency ambulance calls, emergency department attendances or urgent care use. There was a $19.3 \%$ reduction in calls to NHS Direct $(95 \% \mathrm{Cl}-24.6 \%$ to $-14.0 \%$ ) and a $2.9 \%$ increase in emergency ambulance incidents ( $95 \% \mathrm{Cl} 1.0 \%$ to $4.8 \%$ ). There was an increase in activity overall in the emergency and urgent care system in each site ranging $4.7-12 \% /$ month and this remained when assuming that NHS 111 will eventually take all NHS Direct and GP out of hours calls. Conclusions: In its first year of operation in four pilot sites NHS 111 did not deliver the expected system benefits of reducing calls to the 999 ambulance service or shifting patients to urgent rather than emergency care. There is potential that this type of service increases overall demand for urgent care.

\section{INTRODUCTION}

ScHARR, University of Sheffield, Sheffield, UK

Correspondence to Ms J Turner; j.turner@sheffield.ac.uk
A consultation by policy makers in England identified that a key frustration in the general population was access to urgent care. ${ }^{1}$ Urgent care is defined as 'the range of

\section{ARTICLE SUMMARY}

Strengths and limitations of this study

- This is the first controlled evaluation of the impact of the NHS 111 service on the emergency and urgent care system in England. This is timely as the service is being rolled out nationally.

- There is limited evidence on the use of nonclinical call handlers to triage requests for urgent care and this study adds to the evidence base.

- Although we conducted a controlled study other system changes made it difficult to isolate the effects of NHS 111 and we were unable to assess the potential impact on in-hours GP services.

responses to people who require or perceive the need for urgent advice, care, treatment or diagnosis'. ' Problems faced by users of emergency and urgent care included a lack of awareness of services available, confusion about which service to access and multiple service contacts for the same episode. ${ }^{2}$ In England in 2000 a national $24 \mathrm{~h}$ telephone line for advice about health problems, National Health Service (NHS) Direct, was established to address similar frustrations. Calls are answered by a non-clinical call handler and assessed by a nurse either immediately or with a later call back. Despite this service the national consultation found that access problems persisted. ${ }^{1}$

NHS 111 was developed as a solution to these problems by offering a telephone service to manage all requests for urgent help ${ }^{3}$ including requests for out-of-hours primary care, urgent problems that may currently be directed to 999 ambulance services and health information and advice. The key differences from NHS Direct are-access through a free-to-call, easily remembered three digit telephone number '111'; calls answered and assessed immediately by a trained non-clinical call handler without waiting or call backs; only some calls assessed by a nurse; and integration of the assessment 
system with services enabling direct referral to, or appointments to be made with, some services at the time of the call.

The expected benefits of NHS 111 were to improve access to urgent care, increase efficiency by directing people to the 'right place first time' including self-care advice, increase satisfaction with urgent care and the NHS generally, and in the longer term reduce unnecessary calls to the 999 emergency ambulance service and so begin to rectify concerns about the inappropriate use of emergency services. ${ }^{4}$

NHS 111 was established in four pilot sites in England in 2010. It is rapidly becoming available nationally and there is international interest in telephone access to urgent care through non-clinical triage. A mixed methods evaluation focusing on processes, outcomes and costs was conducted in the four pilot sites. We report here on the specific outcome of NHS 111 improving efficiency of service use across the emergency and urgent care system by shifting care from emergency to urgent services. The objective was to assess the impact of NHS 111 on the emergency and urgent care system by examining demand for other urgent and emergency care services to detect if there was any change in how services were used.

\section{METHODS}

\section{Setting and service}

Pilot services were established in four geographical areas defined by primary care trusts, the healthcare commissioning organisations operating in England in 2010. Durham and Darlington is an urban area with a population of around 606000 ; Nottingham is a city of around 300000 with a large minority ethnic population; Luton is a city of around 200000 with a large minority ethnic population; and Lincolnshire is a largely rural area with a city, of population 700000 . The four sites were chosen by the English Department of Health following a request for expressions of interest from commissioning organisations already planning or considering changing telephone access to urgent care. Call handling was provided by an ambulance service in one site and NHS Direct in three sites. In all sites NHS 111 could be accessed directly by dialling ' 111 ' or indirectly where general practice (GP) out-of-hours call-handling services were routed to NHS 111. Calls to NHS 111 are answered and assessed by trained non-clinical call handlers using the NHS Pathways assessment system. ${ }^{5}$ If needed, calls can be transferred for additional assessment and advice from an onsite trained nurse. At the end of the assessment callers are matched to the most appropriate service available at the time of their call from a range of services within the callers' locality using an electronic directory of services linked to the assessment system. This can include emergency ambulance, emergency department (ED), urgent care centre, walk-in centre, minor injury unit, GP out-of-hours service, in hours GP, community services or home care. Referrals can be made to some services by NHS 111 at the time of the call, for example, direct dispatch of an emergency ambulance, appointment booking and transfer of the call to another telephone-based service. A description of the NHS 111 service is provided as a online supplementary file $(\mathrm{S} 1)$.

\section{Design}

The design of this part of the evaluation was a descriptive study of NHS 111 service use and a controlled before and after study using a time series analysis of routine service activity data. Control sites were selected to match equivalent geographical areas to the pilot sites using a two stage process: (1) potential sites were identified by primary care trust area type (county or city), urban/rural mix and same Strategic Health Authority (SHA) or nearest neighbour; (2) from 12 potential sites the final choice was made after matching for a range of 18 criteria based on population demographics, lifestyle, health profile and health service use. A table listing all criteria is provided as a online supplementary file (S2). It was important that control sites had no plans to introduce NHS 111 or make major changes to their emergency and urgent care system in the time frame of the evaluation. We identified three suitable control sites: North of Tyne, Leicester and Norfolk. Leicester was the best match for two pilot sites (Nottingham and Luton). The control site for Luton is not in the same SHA but was the best match for all other criteria and was the only suitable nearest neighbour SHA. For the main impact analysis sites have been combined to provide single pilot and control sites. The characteristics of the pilot and control sites are presented in table 1. For the analyses reported here data from all pilot sites were combined and compared with data from all control sites. Randomisation of sites to be pilots or controls was not possible because the four pilot sites were preselected by the Department of Health.

The four pilot sites became fully operational at different times from July to December 2010. The study periods used were the first full year of operation of NHS 111 and the corresponding 2 years prior to the service starting. During the course of the evaluation NHS Direct continued to operate as a national service within the pilot site areas.

\section{Participants}

Participants were users of the emergency and urgent care systems in the seven pilot and control sites recorded in routine service activity data as having accessed and used a range of emergency or urgent care services during the study periods.

\section{Data collection}

Use of NHS 111

A minimum data set (MDS) was created by the English Department of Health to provide information on NHS 


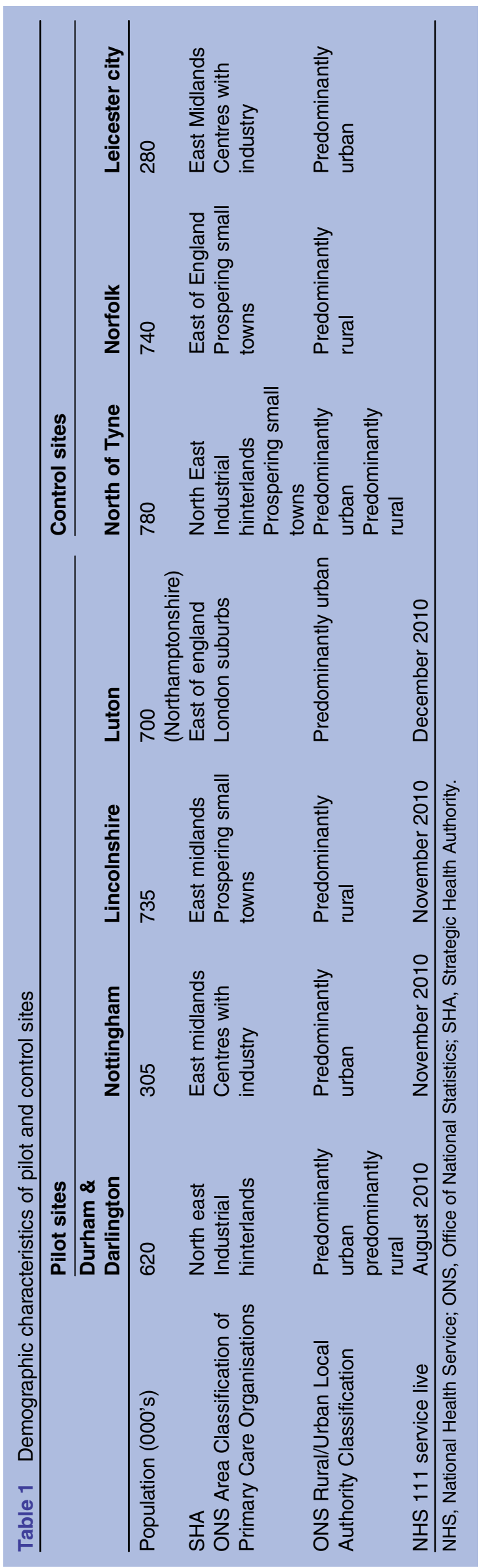

111 service operation. All NHS 111 services submit monthly data and an MDS of activity for combined and individual services is published each month by the Department of Health. ${ }^{6}$ The MDS records activity including numbers of: calls to the service; abandoned calls; triaged calls and transfer times for calls requiring clinical advice from a nurse. The dispositions of NHS 111 calls are recorded in five main categories: (1) ambulance dispatches; (2) recommended to attend ED; (3) recommended to attend primary and community care (including GP, urgent care, dental and pharmacy services); (4) recommended to attend another service (including community nursing, midwives, social services, opticians); (5) not recommended to attend a service (including home or self care and health information). We used the monthly routine MDS counts for each pilot site for the first year of operation to describe call volumes, numbers and proportions of triaged calls and calls passed for nurse assessment and the disposition arrived at following NHS Pathways assessment.

\section{Routine data on use of key services}

NHS 111 had the potential to produce an impact on activity across a range of emergency and urgent care services: calls to the emergency ambulance service; ambulance incidents, that is, an ambulance is sent and arrives at the scene of the emergency incident; ED attendances; contacts with urgent care services such as GP out of hours, urgent care centres, walk in centres or minor injury units; the telephone triage service NHS Direct; same day general practice attendances; and a range of community services such as district nursing, dentists and pharmacies. Data are routinely available for ambulance calls and incidents ED, urgent care and NHS Direct by residents in the seven geographical areas-four pilot and three control sites-for 24 months prior to the start of NHS 111 (2008-2010) and the same data plus calls to NHS 111 in the pilot areas for 12 months after (20102011). Owing to a lack of data availability for separate urgent care services, we had to combine data for out-of-hours primary care contacts, walk-in centre attendances and urgent care centre attendances. The sources of this data were NHS data collections (secondary users service and weekly situation reports) and local management information reports provided by the study sites. For local management information reports a set of data items and definitions was used to standardise data collection across all sites. In one pilot area data on one urgent care contact data item was missing and therefore inputted from the previous and subsequent 3 months activity. All data were collected and collated by the Department of Health commissioning analysis and intelligence team.

We also needed to account for changes to services in the emergency and urgent care system other than NHS 111 occurring in the 36 months. NHS 111 leads and control site evaluation contacts were asked to provide details of changes to emergency and urgent care services occurring in study sites during the study time periods. 
We also searched primary care trust annual reports for 2009/2010 and 2010/2011 for each study site to identify any reported major changes to the emergency and urgent care system.

\section{Analysis}

For the service use analysis we fitted a time series regression model ${ }^{7}$ to the combined pilot site counts to test for preliminary evidence that service use had changed over time. This model consisted of a month effect to help explain variation due to seasonal fluctuations, an overall trend, a before and after step term for other potentially significant changes introduced into the pilot site, and a term for before and after the time when NHS 111 was launched. This was the pilot only model.

We then tested for changes in the pilot sites compared with the control sites using time series regression to test for the impact of NHS 111. We used a simple model with three main elements: (1) the basic model, consisting of a linear time trend in activity over the 36 months constrained to be the same in the pilot and control sites, plus a seasonal effect and a site effect; (2) site specific before and after terms to allow for effects of potentially significant service changes other than NHS 111 introduced during the 36 months, for example, relocation of an ED; (3) a term for the regression of the monthly activity counts on the volume of NHS 111 calls that were triaged that month (the 'dose'). By definition the dose is 0 for all months in the control sites until the launch of NHS 111 in the pilot sites. This regression allowed us to directly estimate the impact of different levels of NHS 111 activity.

We used the regression coefficient to estimate the impact on monthly service use per thousand NHS 111 calls for the pilot sites. The models were fitted and coefficients and SEs estimated assuming normal errors with constant variance in the monthly activity counts. To check the assumption of constant variance, we also fitted models to the square root of the counts which helps stabilise the variance. This produced no important differences in fit so results using the raw count models are reported here as the pilot versus control model. We used the Prais-Winsten procedure in STATA V.12 to fit the time series regression models. ${ }^{8}$ We considered the potential impact of NHS 111 on overall demand for the emergency and urgent care system as this adds an extra service and can potentially add an extra contact if it does not reduce use of other services in the system. We used the routine data to measure monthly use of the different services in each pilot site before and after the introduction of NHS 111 and hence overall use of the emergency and urgent care system. We have then estimated the effect on the system taking two additional factors into account (1) in the 'after' period NHS Direct and NHS 111 were operating concurrently so we have estimated the effect if all NHS Direct calls are taken by NHS 111 and (2) in the 'before' period GP out-of-hours calls (rather than contacts) were taken by a number of providers so we have estimated the number of these calls using the routine NHS 111 data on calls diverted from out-of-hours providers. The assumption of all NHS Direct and out-of-hours calls being managed by NHS 111 reflects the intended national service.

\section{RESULTS}

\section{Use of NHS 111}

In the first year of operation over 400000 calls were made to NHS 111 (table 2). Two-thirds were direct dial of the telephone number ' 111 ' by members of the general public and the others were routed to the service from GP out-of-hours services. Of the calls $22 \%$ were unanswerable because the caller hung up within $30 \mathrm{~s}$. These calls were predominantly not intended for NHS 111 but were from people calling their general practice in the morning to make an appointment with their GP before the out-of-hours rerouting mechanism was switched off. Of the answerable calls $98 \%$ were answered and $277163(78 \%)$ of these were triaged by a call handler using the NHS Pathways assessment system. Reasons for not triaging calls were that the caller hung up, the call was transferred without triage (eg, for a 999 ambulance), or health information only was given. The annual rate of triaged calls/1000 population was 154 . All pilot sites met national quality requirements for call abandonment rates of no more than $5 \%$, and $95 \%$ of calls answered within $60 \mathrm{~s}$. Of the calls $28 \%$ were transferred to a nurse for clinical advice although transfers were lower in the ambulance service provided site $(21.3 \%)$ than in the NHS Direct provided sites (27.9 $33.7 \%$ ). For all sites combined, over half of triaged calls were assessed as requiring primary or urgent care, that is, GP practice, GP out of hours, walk-in centres, urgent care centres, minor injury units, dental service or pharmacist (figure 1).

\section{Impact on emergency and urgent care services}

Across all pilot and control sites there were 13 other system changes reported that were taken into account in the analysis including the opening, closing and relocation of urgent care and walk-in centres, relocation of an $\mathrm{ED}$, attendance reduction schemes, ambulance service conveyance direct to walk-in centres and related publicity campaigns.

Following the introduction of NHS 111, in individual pilot sites there was a statistically significant reduction in urgent care attendances in one site; reduction in calls to NHS Direct in three sites; reduction in ambulance emergency calls in one site and increase in one site, and an increase in ambulance incidents in one site.

For all sites combined, overall, there was no change in three of the five services measured that could be attributed to NHS 111 (table 3). There was a large and statistically significant reduction in calls to NHS Direct of 102 fewer NHS Direct calls per triaged 1000 calls to NHS 111 equating to a $19.3 \%$ (95\% CI $-24.6 \%$ to $-14.0 \%$ ) 
Table 2 Total numbers of NHS 111 calls received, answered, triaged and transferred for nurse assessment in 1 year

\begin{tabular}{|c|c|c|c|c|c|}
\hline & $\begin{array}{l}\text { Durham and } \\
\text { Darlington }\end{array}$ & $\begin{array}{l}\text { Nottingham } \\
\text { city }\end{array}$ & Lincolnshire & Luton & $\begin{array}{l}\text { All NHS } \\
111 \text { sites }\end{array}$ \\
\hline Population covered & 606800 & 300800 & 700300 & 194300 & 1802200 \\
\hline Total number of calls connected to 111 & 209633 & 58397 & 102611 & 38210 & 408851 \\
\hline Direct dial $111 \mathrm{n}(\%)$ & $106961(51)$ & $18354(31.4)$ & $102611(100)$ & 23264 (60.8) & $251190(61.4)$ \\
\hline Switched from other sources n (\%) & $102672(49)$ & $40043(68.6)$ & 0 & 14946 (39.2) & 157661 (38.6) \\
\hline Answerable calls $\mathrm{n}(\%)$ & $165355(78.9)$ & $56539(96.8)$ & $100144(97.6)$ & 37497 (98.1) & 359535 (87.9) \\
\hline Answered calls $n$ ( $\%$ of answerable calls) & $161082(97.4)$ & 55564 (98.2) & 99381 (99.2) & $37073(98.8)$ & $353100(98.2)$ \\
\hline Triaged calls $n$ ( $\%$ of answered calls) & $114686(71.2)$ & 44937 (80.9) & $85509(86.0)$ & 32031 (86.4) & $277163(78.5)$ \\
\hline Transferred to nurse $\mathrm{n}$ ( $\%$ of triaged calls) & $24488(21.3)$ & $13261(29.5)$ & 28871 (33.7) & 10779 (33.6) & $77399(27.9)$ \\
\hline Triaged calls per year per 1000 people & 189 & 150 & 122 & 165 & 154 \\
\hline
\end{tabular}

reduction in monthly NHS Direct activity. There was also a small and statistically significant increase in numbers of ambulance incidents of an extra 24 ambulance incidents/1000 triaged calls to NHS 111 equating to an increase of $2.9 \%$ (95\% CI $1.0 \%$ to $4.8 \%$ ) in monthly ambulance activity.

The counts of contacts with all services in the pilot sites shows that monthly use of the established services in the system slightly increased or slightly decreased, depending on the site but when NHS 111 use was added in, there was an increase in activity overall in every site. When taking in to account the assumption that in the future all NHS Direct and all GP out-of-hours calls will

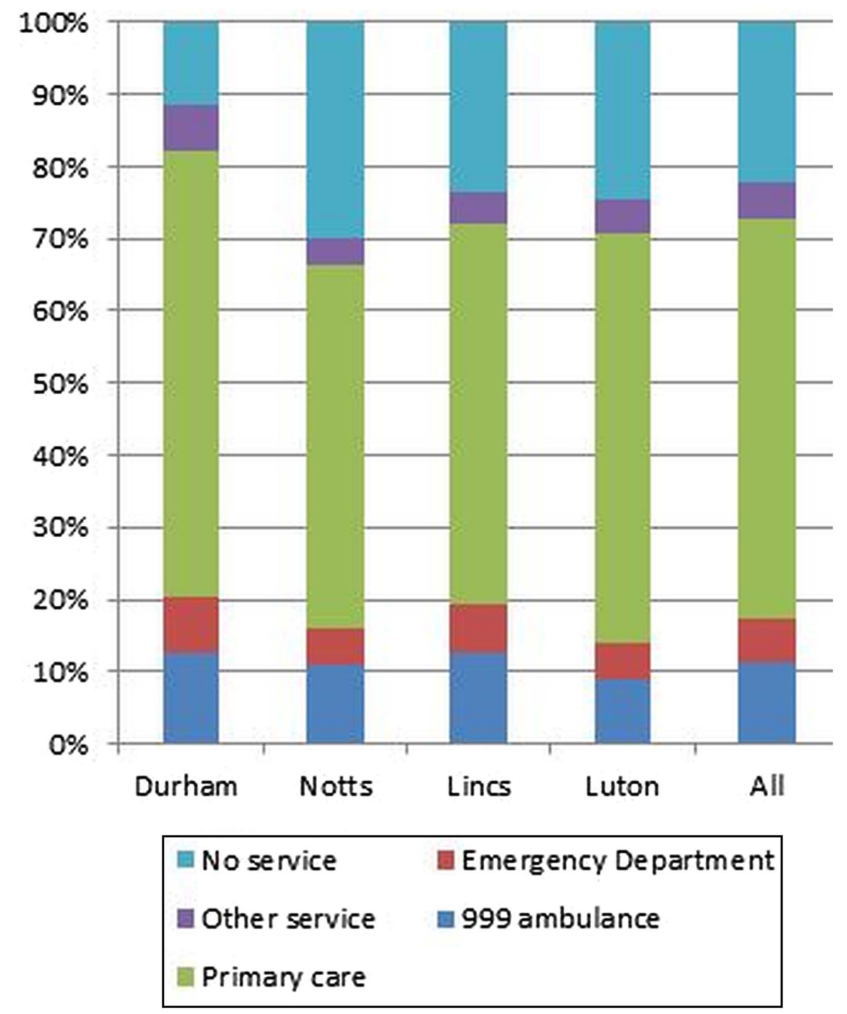

Figure 1 Percentage of triaged, National Health Service (NHS) 111 calls allocated to each emergency and urgent care service in four pilots sites in first year of operation. be directed to NHS 111 this increase, ranging 4.7-12\%/ month, remained (table 4).

\section{DISCUSSION}

\section{Summary of findings}

In the first year of operation NHS 111 pilot sites triaged almost 300000 calls, $72 \%$ of these calls were managed by non-clincial call handlers and just over half of the calls were directed to primary care. However, there was no evidence that NHS 111 changed use of most of the emergency and urgent care services it was possible to measure. There was a large reduction in use of NHS Direct as calls transferred to NHS 111 but an increase in numbers of emergency ambulances sent to patients and there is potential that overall demand for services across the emergency and urgent care system could increase.

\section{Context of other evidence}

Policy makers in England established the first national telephone triage service in the world-NHS Direct-and there was considerable international interest in this service as well as the evaluation of its pilot. ${ }^{9}$ A Cochrane systematic review of the impact of telephone triage services identified that little research had been undertaken on the effect of these telephone services on emergency services. ${ }^{10}$ The lack of impact of NHS 111 on ED attendances replicates the findings from the earlier evaluation of NHS Direct pilots. ${ }^{11}$ The increase in ambulance incidents found in our study was not found for NHS Direct pilots. There is some evidence that telephone triage can reduce the use of general practice and general practice out-of-hours. ${ }^{10} 11$ A lack of routine data available for daytime general practice services in our study means we were unable to assess the impact of NHS 111 on use of general practice.

A key feature of NHS 111 is the use of non-clinical call handlers to assess calls. A systematic review of appropriateness of and compliance with telephone triage ${ }^{12}$ found only two papers on non-clinical triages and these were of little relevance to NHS 111 as no assessment software was used. 
Table 3 Summary of estimated effects of NHS 111 on other emergency and urgent care services: percentage of change in monthly activity counts

\begin{tabular}{|c|c|c|}
\hline Service activity & $\begin{array}{l}\text { Pilot only model-estimated change in monthly } \\
\text { service activity per } 1000 \text { triaged NHS } 111 \text { calls } \\
\text { after the introduction of NHS } 111\end{array}$ & $\begin{array}{l}\text { Pilot vs control model-estimated } \\
\text { percentage of change in monthly activity } \\
\text { ( } 95 \% \mathrm{Cl} \text { )in pilot sites compared to } \\
\text { control sites after the introduction of } \\
\text { NHS } 111\end{array}$ \\
\hline ED attendances & $-1(-66,+64)$ fewer attendances & $-0.1(-3.8$ to +3.7$)$ \\
\hline $\begin{array}{l}\text { GPOOH, WiC, UCC. } \\
\text { MIU attendances }\end{array}$ & $+47(-66,+159)$ extra attendances & $+2.5 \%(-3.5$ to +8.5$)$ \\
\hline Calls to NHS Direct & $-102(-130,-74)$ fewer calls & $-19.3 \%(-24.6$ to -14.0$)$ \\
\hline $\begin{array}{l}\text { Calls to } 999 \text { ambulance } \\
\text { service }\end{array}$ & $+3(-31,+37)$ more calls & $+0.3 \%(-3.1$ to +3.7$)$ \\
\hline $\begin{array}{l}\text { Ambulance } 999 \\
\text { incidents where an } \\
\text { ambulance arrives at } \\
\text { the incident scene }\end{array}$ & $+24(+8,+39)$ more incidents & $+2.9 \%(+1.0$ to +4.8$)$ \\
\hline
\end{tabular}

\section{Strengths and limitations}

This evaluation has three strengths. First, there is little research evidence about telephone triage services operated by non-clinical call handlers, and the impact of telephone triage services on use of the emergency and urgent care system, making this evaluation of NHS 111 a valuable addition to the evidence base. Second, the evaluation is timely given that NHS 111 was established in pilot status in 2010 and is being rolled out nationally in England during 2012/ 2013. Third, it is a large controlled study that has included data from a population of 3.6 million people over 36 months on the use of five services as well as NHS 111. The evaluation has three limitations. First, there was considerable 'noise' in the analysis of impact on services in terms of changes made to the range of services in the emergency and urgent care system other than NHS 111 in the pilot as well as control sites. We recorded 13 different system changes across the pilot and control sites. This made it challenging to detect the effect of NHS 111 but the time series analysis was a sophisticated approach to deal with these difficulties. Second, there was no routine data available for a key service that may have been impacted by NHS 111: day time general practice, so the effects on this part of the system remain unknown. Finally, the timing of policy evaluations must be balanced not only to ensure early feedback to policy makers but also to allow for a service to become established. This evaluation is based on the first year of operation of a new service and so while early lessons are valuable the impact may change as the service matures and develops.

\section{Implications}

The findings of this study raise five key questions for the development of a national service. First, the four NHS 111 pilots did not produce some of the key expected benefits in their first year of operation. In all four pilot sites there was an increase in emergency ambulance incidents compared to controls and this was statistically significant in one service and for all services combined. The benefit expected was a reduction in use of this service in the longer term. In 2011/2012 ambulance services in England attended 6.71 million incidents ${ }^{13}$ and the $2.9 \%$ increase in ambulance incidents we have estimated could potentially result in an additional 195000 annual attendances nationally or about 14500 extra attendances for an ambulance service attending 500000 incidents/year. It is important to further investigate and understand how the assessment system triages calls to the ambulance service in order to avoid unnecessary use of emergency ambulances.

Second, all four pilot sites used the same call assessment system-NHS Pathways-to manage calls to NHS 111. This means the findings reflect the inherent characteristics of the NHS Pathways system such as the levels of caution and risk built into the assessment algorithms, particularly as it is designed to be used by non-clinical call handlers. There may be less flexibility to change decisions compared with assessments made by nurses ${ }^{14}$ and it is possible that a different call assessment system could produce different results.

Third, during our evaluation NHS Direct was still running as an alternative service. The policy plan is that NHS 111 will replace NHS Direct and there are significant implications to this strategy. NHS Direct was established to direct people to the right place but also in practice offers advice to people who do not need contact with a service. The emphasis of NHS 111 is on direction to right place rather than reassurance and self-care advice. In our evaluation, NHS 111 managed predominantly out-of-hours calls for urgent healthcare. If current callers to NHS Direct are shifted to NHS 111 the call volumes may increase substantially, the characteristics of the population using the service may change and consideration will need to be given to how the principles of NHS 111 in terms of immediate access without waiting, particularly for clinical advice, can be sustained. 


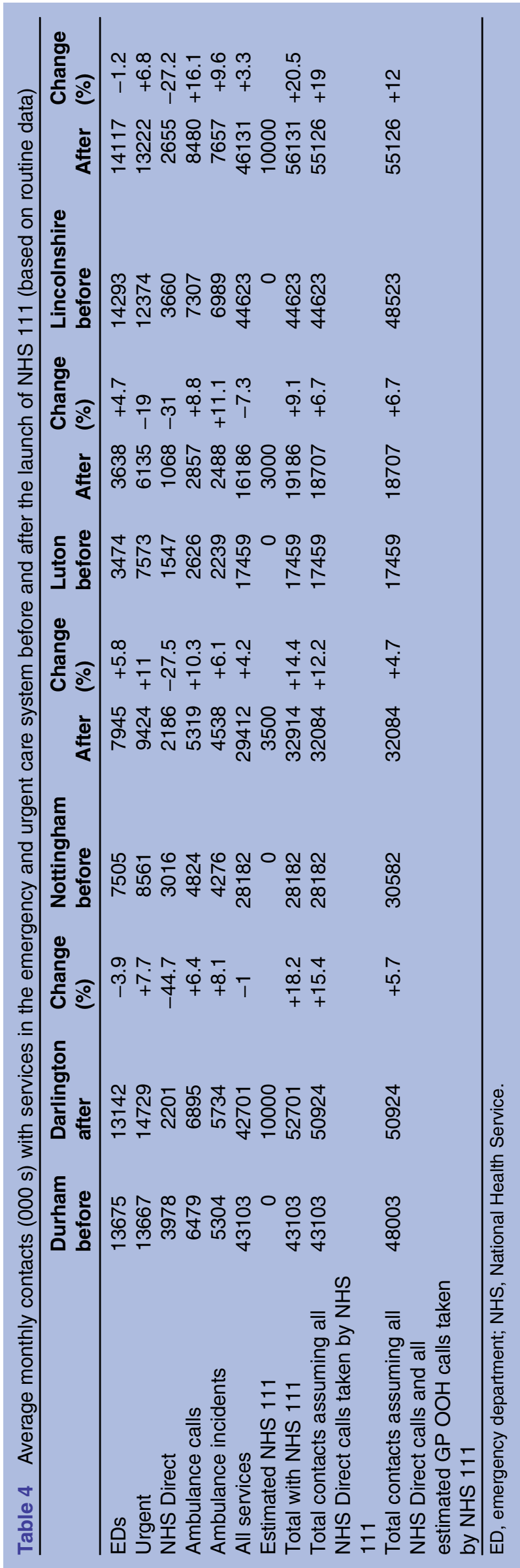

Fourth, another important question to consider is whether the introduction of NHS 111 is creating supplier induced demand and therefore increasing overall demand for emergency and urgent care. There was some evidence from our system impact analysis that emergency and urgent care service use had increased overall but we cannot say if this is a real increase in demand or a shift from in-hours GP services. It is possible that, once NHS 111 is a national service with a higher profile, demand for the service could change either by generating new demand or by people using it as an alternative to in-hours primary care, or a combination of both.

Finally, it is useful to reflect on the expectations of the service. The provision of a telephone service which quickly guides people needing urgent care advice to the most appropriate service is sensible given repeatedly expressed concerns by the general public about confusion around which service to access when needing urgent care. Key aspects of the service such as an easy-to-remember number, emphasis on fast triage and smooth transfer to the 'right service, first time' are desired by the general public. In our evaluation we found that alongside implementation of NHS 111 there were various reorganisations of services and implementation of demand management schemes in the pilot as well as control sites. It is probably unrealistic to expect any one service, such as NHS 111, to do everything and real improvements may only be gained when a series of coordinated measures designed to increase efficiency across all services are implemented.

Acknowledgements The authors would like to thank Professor Simon Dixon for advice about the economic analysis; Neil Shephard for statistical help with the system impact analysis and Dr Claire Ginn for obtaining the routine data used in the impact analysis.

Contributors JT and AOC conceived the study and designed it with help from JN and EK. JN conducted the system impact analysis. JT wrote the first draft of the paper. All authors assisted in the interpretation of data and revising the paper and approved the final draft. JT is the guarantor.

Funding The study was funded as an independent research project by the policy research programme in the Department of Health Grant number 0490016. The Department of Health contributed to the development of the research questions to be addressed.

Competing interests The Department of Health policy research programme provided grant funding to the Medical Care Research Unit, University of Sheffield. AOC and JN are co-applicants on a research grant with NHS Direct studying tele-health for people with long term conditions. A family member of AOC won a contract to offer patient feedback for NHS 111 sites in London in June 2012

Ethics approval The study was approved by the Leeds (Central) Research Ethics Committee Reference number 10/H1313/57. NIHR CRN study ID: 9275

Provenance and peer review Not commissioned; externally peer reviewed.

Data sharing statement $A$ dataset of aggregated monthly contact counts for five emergency and urgent care services in seven sites for 36 months is available on request from the corresponding author at j.turner@sheffield.ac. uk, the Department of Health publish monthly open access activity data for NHS 111 services available at http://transparency.dh.gov.uk/category/statistics/ nhs-111-statistics/

Disclaimer This is an independent report commissioned and funded by the Policy Research Programme in the Department of Health. The views expressed are not necessarily those of the Department. 
Open Access This is an Open Access article distributed in accordance with the Creative Commons Attribution Non Commercial (CC BY-NC 3.0) license, which permits others to distribute, remix, adapt, build upon this work noncommercially, and license their derivative works on different terms, provided the original work is properly cited and the use is non-commercial. See: http:// creativecommons.org/licenses/by-nc/3.0/

\section{REFERENCES}

1. Department of Health. Direction of travel for urgent care. 2006. http:// webarchive.nationalarchives.gov.uk/+/www.dh.gov.uk/en/ consultations/Liveconsultations/DH_4139428

2. O'Cathain A, Coleman P, Nicholl J. Characteristics of the emergency and urgent care system important to patients: a qualitative study. J Health Serv Res Policy 2008;2:19-25.

3. Department of Health. High quality care for all: NHS next stage review final report. 2008. http://www.dh.gov.uk/en/Publicationsandstatistics/ Publications/PublicationsPolicyAndGuidance/DH 085825

4. NHS Connecting for Health. Introduction to NHS 111. 2011. http:// www.connectingforhealth.nhs.uk/systemsandservices/pathways/ news/nhs111intro.pdf

5. NHS Connecting for Health. NHS Pathways care narrative. 2012. http://www.connectingforhealth.nhs.uk/systemsandservices/ pathways/comms/corenav.pdf
6. Department of Health. NHS 111 statistics. http://transparency.dh.gov. uk/category/statistics/nhs-111-statistics

7. Campbell MJ. Time Series Regression. In: Encyclopedia of Biostatistics. Armitage P, Colton T. Chichester: John Wiley \& Sons, Ltd, 1998. (ISBN 0471 975761).

8. StataCorp. Stata: Release 12. Statistical software. College Station, TX: StataCorp LP, 2011.

9. Munro J, Nicholl J, O'Cathain A, et al. Evaluation of NHS direct first wave sites. Final report of the phase I research. Medical care research unit. University of Sheffield, 2001.

10. Bunn F, Byrne G, Kendall S. The effects of telephone consultation and triage on healthcare use and patient satisfaction: a systematic review. Br J Gen Pract 2005;55:956-61.

11. Munro JF, Nicholl JP, O'Cathain A, et al. Impact of NHS Direct on demand for immediate care: observational study. $\mathrm{Br}$ Med $\mathrm{J}$ 2000;321:150-3.

12. Blank L, Coster J, O'Cathain A, et al. The appropriateness of, and compliance with, telephone triage decisions: a systematic review and narrative synthesis. J Adv Nurs 2012;68:2610-21.

13. NHS Information Centre. Ambulance Services-2011/2012. NHS Information Centre, 2012. http://www.ic.nhs.uk/statistics-and-datacollections/audits-and-performance/ambulance/ambulance-servicesengland-2011-12

14. Snooks $\mathrm{H}$, Griffiths $\mathrm{L}$, Peconi J, et al. Real Nursing? The development of telenursing. J Adv Nurs 2008;61:631-40. 